\title{
AN INTEGER LINEAR PROGRAMMING FORMULATION FOR POST-DEPARTURE AIR TRAFFIC FLOW MANAGEMENT
}

\author{
Miriam Bongo and Charlle Sy \\ Department of Industrial Engineering, Gokongwei College of Engineering, De La Salle University, Manila, \\ Philippines, Tel: +63 (02) 8524 4611, e-mail: miriam_bongo@dlsu.edu.ph, charlle.sy@dlsu.edu.ph
}

Received Date: March 16, 2020; Revised Date: September 20, 2020; Acceptance Date: December 16, 2020

\begin{abstract}
The air transportation domain faces issues in air traffic congestion which leads to delays affecting a network of flights. As stakeholders strive to address such issue by applying air traffic flow management (ATFM) actions, there exists an apparent divide in the solution objective and perspectives. In the extant literature, solution approaches involving ATFM actions are often framed from the perspective of only one stakeholder to another. Such a scheme does not comprehensively cover the overall goal of the stakeholders, thus, provides inadequate, even conflicting, solutions. Therefore, this paper proposes an integer linear programming model for a rerouting problem that satisfactorily incorporates the individual interests of stakeholders (i.e., airport management, airline sector, air traffic management) in the commercial aviation industry and the common goal of ensuring safety in flight operations. The proposed model is designed to tactically select an alternate route when the primary route is constrained due to uncertainties such as inclement weather in a post-departure scenario. A hypothetical case study involving multiple destinations and alternate routes is carried out to illustrate the validity of the model. A Demo version of Lingo software is used to run the proposed model. Notable computational results show significant differences of selected routes as individual system interests are taken into isolation compared to when the general, collaborative model is implemented. In other words, the proposed model is able to show that preferences in alternate routes do vary with the individual interests of stakeholders, more so with the integration of the collaborative decision among stakeholders. Therefore, this research work provides a groundwork to a more comprehensive take of managing air traffic scenario involving all phases of flights. This is realized by providing a proof that significant shifts of decision solutions occur when the overall goal of stakeholders is considered rather than taking their individual interests into isolation.
\end{abstract}

Keywords: Air traffic flow management, Collaborative decision-making, Integer linear programming, Post-departure, Rerouting

\section{Introduction}

Air traffic congestion has long been a pressing concern in the domain of air transportation industry as a result of the increasing complexity of airport network structure, its operational environment, and aircraft ground movement [1]. Such an issue further cuts across operational concerns on huge credit losses, poor air passenger satisfaction, low air quality, and increased emissions, among others. As a result, implementing air traffic flow management (ATFM) actions such as ground holding, airborne holding, rerouting, and speed controlling, are pushed forward. This is done to smooth the air traffic flow at an optimal rate while not exceeding the capacities of airports and other air traffic control facilities [2]. In some significant cases when an aircraft is already in its post-departure phase upon detected resource unavailability, or in other instances the occurrence of convective weather in supposed airspace sectors, rerouting is believed by the stakeholders of the commercial 
aviation industry to be the most suitable recourse based on considerations in safety, the general welfare of the entire transportation system, and congestion costs [3].

Rerouting is characterized by a deviation from the initially planned routes, which is deemed necessary depending on some cases [4]. If the original route traverses a region that needs to be avoided due to poor weather conditions, sector congestion, or potential conflict with another aircraft, a reactive rerouting is performed. On the other hand, opportunistic rerouting is undertaken when there is lower traffic than expected, improved weather conditions, and reduced uncertainty. Such an option allows for a more direct path than the one previously defined in the flight plan to save significant flight time and other related costs. Any of these rerouting schemes may be implemented prior to departure or after the aircraft has taken off. It is imperative to note, however, that rerouting of flights depends on the collaborative decision-making between the airlines and air navigation services such that these stakeholders' interests are well-satisfied such as performance characteristics, for instance $[5,6]$.

Due to the complexity and the dynamic nature of the decision-making involved in rerouting of flights as a significant measure to aid in ATFM, several thematic analyses have widely tackled this area in conjunction with other ATFM actions (e.g., ground holding, airborne holding, and speed controlling) in the air transportation domain. The earliest work can be traced back from the work of Bertsimas and Stock Patterson [7] involving a 0-1 integer programming model for the deterministic multiairport traffic flow management program, which includes both ground and airborne delays. Model variations, for one, on the rerouting of aircraft, are also developed to account for dynamic rerouting decisions via path approach and sector approach. Using a different method, that is, an aggregate model, Bertsimas and Patterson [8] later addressed the same problem on dynamically rerouting aircraft by formulating a dynamic, multicommodity, integer network flow model.

However, it is then found by Bertsimas et al. [9] that the computational performance of this model is unable to address realistic, very large-scale instances. Therefore, a mathematical model is proposed combining the flexibility of the range of available ATFM actions previously presented by Bertsimas and Patterson [8] and the powerful mathematical properties of the model introduced by Bertsimas and Patterson [7] in order to handle large-scale problems. It is imperative to note that in this model, the trade-off between airport arrival and departure capacities and the aspect of fairness among airlines are adequately reflected as constraints such that compliance to some general principles may be observed accordingly. In fact, Zhang et al. [10] also emphasized the need for creating a strategy that involves effectiveness and fairness among stakeholders of the commercial aviation industry. This, in turn, led to a formulation of another version of a mathematical model consisting of the fairness principle.

However, it is argued that to take the concept of fairness among airlines as a constraint in a mathematical model can neither achieve the desired fairness nor satisfy the needs of efficiency. Under such a premise, an air traffic flow network rerouting problem (ATFNRP) which is multi-objective in light of minimizing total delay cost and maximizing fairness among airlines at the same time is developed. In specific, a Multi-Objective Evolutionary Algorithm based on Decomposition (MOEA/D) is used to further address ATFNRP via a proposed multi-objective model. Similarly, García-Heredia et al. [11] took the fair distribution of delays between different flights as one of the coefficients defined in the objective function of a combinatorial optimization model for the ATFMRP. It is contended that the proposed model is flexible to fixing coefficients in the objective function such that corresponding considerations in the ATFMRP environment is well-accounted for, 
unlike Bertsimas et al.'s [9] and Agustín et al.'s [12] prior models. Note that Agustín et al. [12] also presented a deterministic mixed 0-1 model for the ATFM problem allowing for flight cancellations and rerouting, whenever deemed necessary, as well as considering nodes instead of arcs in flight paths.

In the literature, previous mathematical models are framed under the goal of minimizing cost which only serves beneficial to the airline sector alone. Considering that the dynamics of air transportation system involves collaboration among stakeholders (i.e., airport, airlines, air traffic service), it is essential to arrive at a solution that not only maximizes the sole interest of a stakeholder but also satisfies the relative goal of the other stakeholders. Furthermore, focusing on the cost implications of managing air traffic appears to be rather unsuitable given that the chief collective concern of the stakeholders is to ensure safe operation of flights from origin to destination $[13,14,15]$. At the en-route phase of a flight, ensuring safety becomes increasingly complex as the air traffic scenario may be confronted with uncertainties in weather.

Therefore, stakeholders operate on tactical planning level involving modifications in the air traffic flow and capacity of sectors including rerouting of flights [16]. With such a case, setting safety as a focal objective in the air traffic management can satisfactorily benefit not only the stakeholders but also the entire air transportation sector. Safety in air transportation has been widely tackled under various focus such as key performance indices (KPIs) of stakeholders [17] as well as assessing safety performance and risk [15, 18, 19, 20, $21,22]$, providing safety performance indicators [14, 23], exploring critical attributes during air traffic congestion [24], and aviation safety metrics [25], among others. Despite the potentially outstanding research efforts of prior works in the literature, a common difficulty of quantifying safety seems to persist due to the very high safety level in the air transportation sector and a consequently low number of incidents and accidents used as a basic aspect for measuring safety [15].

Addressing the significant limitation in the literature of considering a singlestakeholder based model for ATFM decision-making, this paper seeks to develop a decision support system based on an integer linear programming model which takes into account the diverse interest of stakeholders concerned mainly in the event of air traffic congestion needing immediate attention on rerouting decisions. The proposed integer linear programming model is patterned after the seminal work of Bertsimas and Stock-Patterson [7] which is able to represent the system-wide scenario of air traffic management involving various ATFM actions. With the use of relevant notations that best represent post-departure aircraft rerouting problem from this seminal model, a comprehensive view of the issue at hand can be achieved such that an alternate route is explicitly selected upon implementation of the model.

In other words, the use of the proposed model provides a straightforward feasible solution on the set of alternate routes that corresponds to the safety objectives under system constraints. Other solution approaches that attempt to address rerouting problem at either a general or specific view, for instance, a dynamic, multicommodity, integer network flow model [8], MOEA/D [10], and a combinatorial optimization model [11] is not able to directly point out an alternate route to take during disruptions. Nevertheless, these models are framed to generally address objectives in total delays and its associated costs. As a contribution to the body of knowledge in the area of air transport management, the proposed model is hinged on the safety objective satisfying the collaborative interests of stakeholders. Such a model representing safety as an objective is a novel application in the air transportation management domain, which typically considers either cost as an objective function or safety measures (e.g., minimum separation distance) as a model constraint. 


\section{The Need for Multi-Objective Decision-Making in a Rerouting Problem}

In the general air traffic congestion context, stakeholders of the commercial aviation industry serve as key decision-makers should unforeseen disturbances occur at any given time during aircraft operations. Other than ground holding, speed controlling, and airborne holding, rerouting is established as a favorable alternative measure of ATFM, especially on instances when the latter measures cannot hold true (i.e., during post-departure operations). These stakeholders strive to reach one common goal of ensuring air traffic congestion while meeting individual auxiliary interests (see Figure 1). Such individual goals may include, but not limited to, economical and efficient manner of aircraft slotting, maximum aircraft utilization, and reduction of more complex workload demands of air traffic service providers [26].

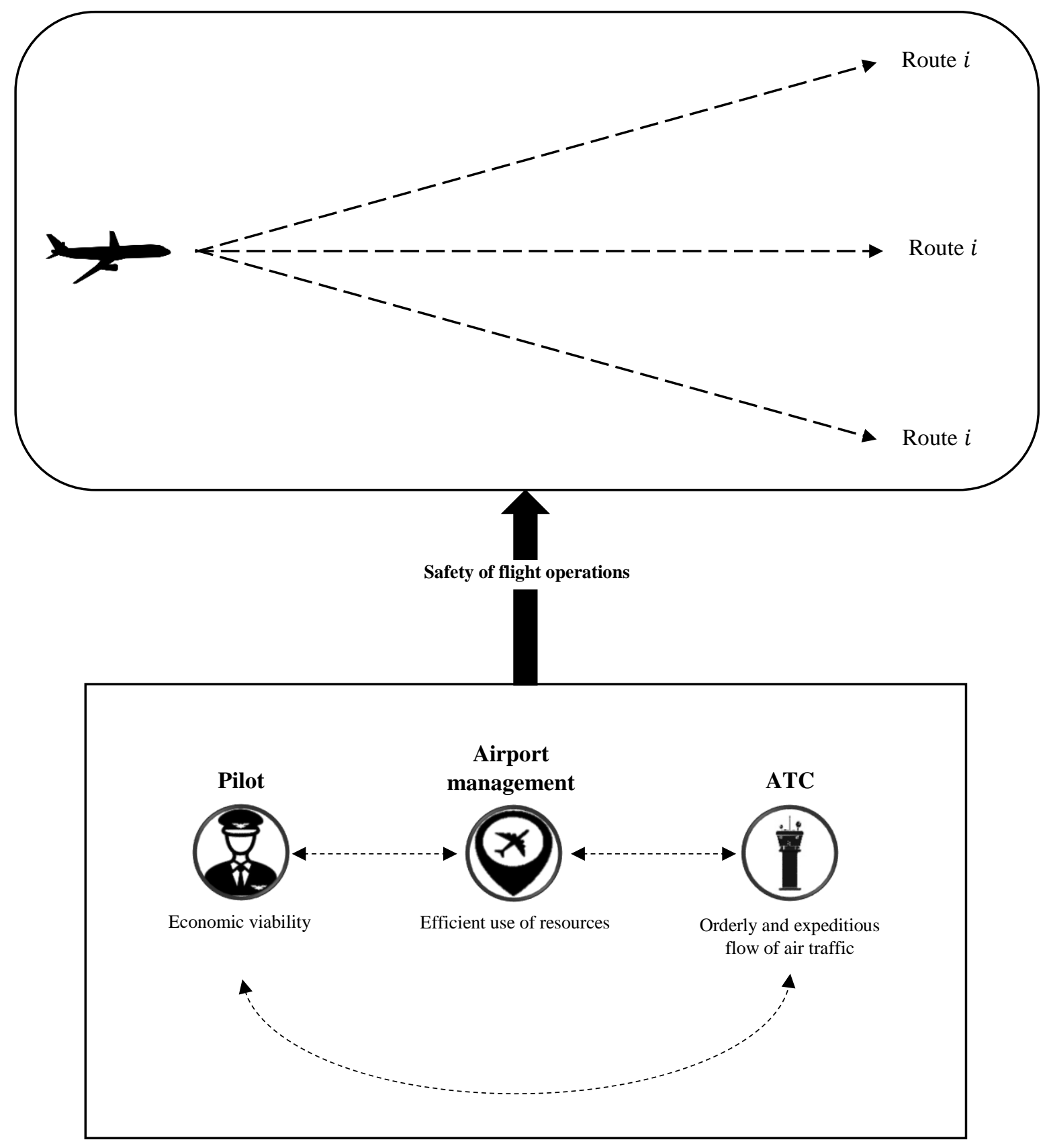

Figure 1. Interaction among stakeholders during rerouting of aircraft 


\section{Airport Management}

While there exists an apparent collaboration between airline companies and air traffic management in the event of disruptions in aircraft operations, the airport management is deemed to be an equally significant stakeholder as it dictates the number of arriving and departing flights an airport can accommodate based on its configurations [27, 28].

Furthermore, airports serve to provide necessary facilities for air traffic management services while closely coordinating with air navigation service providers on matters relating to operations and de-saturation of airports [29, 30]. Due to the uncertainty associated with the occurrence of inclement weather, airport managers are faced with the challenge of sufficiently creating decisions, with limited information, that shall handle imbalances between resource capacity and air traffic demand [31]. Weather conditions are considered as a key driver to airport capacity as it influences the selection of runway configurations and its throughput performance [32]. While changes in the air traffic management services, specifically in en-route services, have relatively less impact on airports, corresponding charges may be incurred to the airport management itself as well as a detriment on the competitiveness of an airport [29]. During real-time conditions involving disturbances in weather, the airport management controls arrival rates into an alternative area away from the constrained one by adding spacing at airspace fixes with the aid of reactionary measures coordinated with air traffic controllers [32]. In terms of collaboration among other stakeholders, while airports provide facilities for use in aircraft operations, an implementation of an ATFM policy may have a rippling effect on the entire transportation system. For instance, a taxi scheduling involving wait-at-gate and free-the-gate implementation [33].

\section{Air Traffic Management}

The air traffic management handles the utilization of airspace resources to support aircraft operations to and from a spatial point [34]. Due to the complexity of managing an airspace resource, the air traffic control facility designates different air traffic service providers that seek to oversee aircraft operations according to a flight's phase. Such designation, although separate in units, is hinged toward the common goal of delivering a safe, orderly, and expeditious flow of air traffic between aircraft [35].

The process of providing air traffic service begins when the pilot requests departure clearance from the origin airport's aerodrome control tower (ADCT). The aircraft on the ground waiting for clearance is under the origin airport's ADCT's jurisdiction until its flight level is at, say, 10 nautical miles. When the request for clearance is granted, the aircraft departs from the gate and taxi-out. Then, the responsibility of monitoring the aircraft is handed over to terminal radar approach control (TRACON) when it reaches beyond 10 nautical miles or until it approaches an altitude of 11,000 feet or above. During the flight's en-route, the control is again handed from one sector to another and from one air route traffic control center (ARTCC) to another when it crosses an airspace boundary. Once the final sector is crossed, the aircraft moves to its descent phase which is 5 to 20 nautical miles from the destination airport and when its altitude is below 10,000 feet. The destination airport's TRACON then controls the aircraft in the terminal airspace during this phase. The pilot then requests for landing clearance from the destination airport's ADCT. At this phase, destination airport's ADCT grants landing clearance as the situation allows and then takes control of the aircraft on the final approach to the receiving airport. The destination airport's ADCT further controls the aircraft on the ground. The same process of requesting for air traffic service is followed after the aircraft's turnaround is completed and is subjected to its next flight leg. 
To some extent of uncertainties at any point of a flight's phase, air traffic service providers coordinate with the airline companies along with the airport management to address any disruption and prevent further damage it may cause (e.g., extended delays). Suppose a system disruption occurs due to inclement weather, flights are recommended to be rerouted, or cancelled, to alternate airports. Then, air traffic service providers coordinate with airport operators to adjust scheduled throughput accordingly while ensuring feasible landing and take-off schedules [36, 37].

\section{Airlines Sector}

Delays in aircraft operations are considered an urgent and essential issue to be tackled under the umbrella of the airline industry as its downstream effect span across various negative effect to the airline's reputation, service quality, crew connections and passenger itineraries, and profitability [38]. While the sources of uncertainties remain to root from uncontrollable variables such as inclement weather, airline companies tend to implement reactive strategies including the rerouting of flights typically designed to be as quick and cost-effective as possible [39]. Such efforts are designed in a way that the goals laid on airline companies are satisfactorily met. These goals include increasing market share, maximizing passenger revenue, and reducing operating costs $[38,40]$. Among these goals, an even more important aspect is valued by the airline industry as in the International Air Transport Association (IATA) principles - safety [41 in 42].

Traditionally, airlines practice competitive scheduling, that is over-scheduling of flights at airport facilities [43], and systematically utilizing smaller aircraft with higher frequency shares to retain market shares [44], the volatility of a flight's travel time is significantly affected, thus, resulting to delays. This happens when an aircraft is permitted to but encounters congested airspace regions or unavailable airport slots. As a result, the aircraft is required to hold in position until a slot becomes available for it to utilize. Otherwise, a ground holding policy is set up before the departure of flights which imposes aircraft to absorb delays on the ground to minimize the cost of delays in the entire network [45]. At a more specific level, airline companies work in close coordination with air traffic management, also known as air navigation service providers, to generate better ATFM measures from both individual and system-level interests [46].

\section{Mathematical Formulation}

Here, a set of flights, each with the corresponding destination, is outlined after the goal of addressing sector congestion under a constrained route by observing safety measures following a collision-free flow of air traffic. That is, maximizing the distance from one flight to another in a given route. By ensuring that the separation distance between flights is maximized, a safe operation of aircraft can be correspondingly achieved. Furthermore, the proposed model focuses on the rerouting decisions given that a destination airport with a predefined primary route has alternate routes for flights to traverse should disturbances in weather be evident at any given period.

\section{The Integer Programming Model for Post-Departure ATFM}

Decision Variables. The decision variables from Bertsimas and Stock-Patterson [7] are used to represent the set of routes an aircraft may traverse to reach its destination. Since postdeparture aircraft are closely considered in this case, no cancellation of flights and ground holding procedures are possible as a priori dictates that each aircraft has already taken-off by the end of a feasible time window; hence, landing within a feasible time window 
determined by the time of departure. Furthermore, two major assumptions are set in the proposed model. First, as aircraft traverse to an alternate route, there is no need to adjust its speed, either slowing down or speeding up, to cover lost travel time or address impending conflict with another aircraft. Second, incoming flights under consideration are assumed to be heading towards the same destination airport without the need to be diverted to an alternate airport. This scenario represents a typical flight sequence that requires rerouting of flights during the post-departure phase. Therefore, the decision variable for the proposed model is represented by the following notation:

$$
w_{j}^{d}= \begin{cases}1, & \text { if a flight going to destination } d \text { selects route } j \\ 0, & \text { otherwise }\end{cases}
$$

Notation: The following notations are considered for the model formulation:

$J \quad \equiv$ set of routes,

$D \quad \equiv$ set of destination points,

$F \quad \equiv$ set of flights,

$c \quad \equiv$ cost of using a route,

$B \quad \equiv$ accumulated budget of operating air carriers,

$R \quad \equiv$ capacity of the route (i.e., the maximum number of aircraft that can be handled by a route),

$E \quad \equiv$ maximum allowable time for the flights to traverse a route without incurring delays,

$m \quad \equiv$ minimum separation distance between aircraft in distance units.

Objective Function: The proposed model aims to maximize the separation distance among flights in a given sector due to the rerouting procedures undertaken on a flight-by-flight basis. The distance is defined by the longitudinal separation between aircraft in the same sector which strictly follows the minimum separation standards specified by the ICAO (see Figure 2). The objective function of the proposed model is motivated by the principle that potential conflict between aircraft can be avoided when the longitudinal distance is maximized. Thus, a conflict-free operation of flights translates to safety.

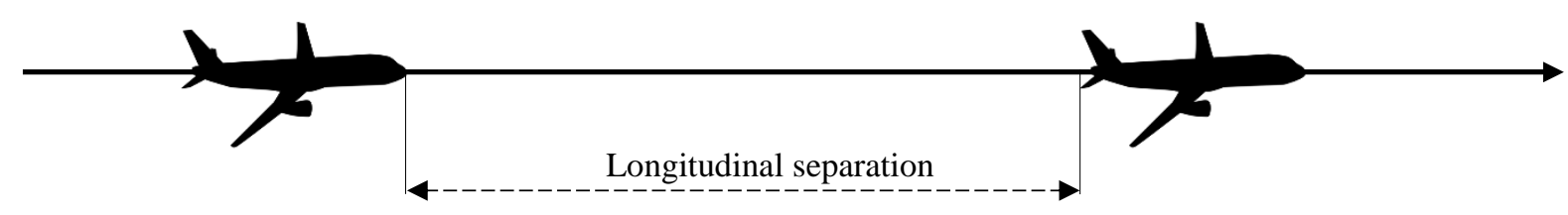

Figure 2. Longitudinal separation between aircraft following minimum separation standards

The objective function is therefore presented as follows:

$$
\text { Maximize } \sum_{d \in D}\left(m_{j}^{d} \cdot w_{j}^{d}\right)
$$


Constraints: The constraints considered in this model are as follows:

$$
\begin{array}{ll}
\sum_{j \in J}\left(f \cdot w_{j}^{d}\right) \leq R_{j} & \forall d \in D \\
\sum_{j \in J}\left(c_{j}^{d} \cdot w_{j}^{d}\right) \leq B & \forall d \in D \\
\sum_{j \in J}\left(r_{j}^{d} \cdot w_{j}^{d}\right) \leq E & \forall d \in D \\
\sum_{j \in J} w_{j}^{d}=1 & \forall j \in J \\
w_{j}^{f d} \in\{0,1\} & \forall f \in \mathcal{F}, j \in J, d \in D
\end{array}
$$

Constraint (1-3) accounts for the individual interests of stakeholders in the context of air traffic management. Specifically, Constraint (1) ensures that the number of flights $f$ considered at a given period as it takes route $j$ shall not exceed the total number of aircraft that can be handled by route $j$. Such a constraint lies in parallel to the immediate concern of airport management on the efficient use of resources including the airspaces. The total number of aircraft allowed to occupy a route is also determined alongside the consideration in minimum separation distance between aircraft. Constraint (2), on the other hand, represents the economic implication of selecting a route concerning the air carriers' discretion. This constraint ensures that the total cost, $c_{j}^{d}$, of selecting a route for each destination point is within the budget allocation $B$ set by the air carriers. The total cost attributed to this constraint corresponds to the cumulative cost of utilizing a flight route, additional fuel consumption, and extra crew cost. Constraint (3) represents the interest of air traffic management to deliver flights to and from a point of reference in an orderly and expeditious manner. This constraint further ensures that flights are within the tolerable and acceptable flight time without incurring considerably huge system-wide delays. Constraint (4) forces the model to select only one alternate route traversed by each flight for each destination point such that if a flight chooses route $j$ for destination $d$, then $w_{j}^{d}$ where $j$ is not the chosen route has to take a numerical value equal to 0. Constraint (5) implies binary variables for $w_{j}^{d}$.

\section{Computational Results and Implications}

The proposed integer linear programming model has been implemented in an ASUS laptop computer under the Windows 10 Home Single Language operating system with Intel Core i7 8th Gen processor and a 16.0 GB RAM. A Demo Lingo/Win64 is used to run the model consisting of 15 decision variables and 31 constraints. As a baseline scenario for this study, a hypothetical data set consisting of 5 aircraft operated by the same air carrier is scheduled to traverse via a primary route established in the flight plan per destination point. Suppose that a restriction is imposed on such primary route, decision-makers in the commercial aviation industry involving the airport management, air carrier, and air traffic management are forced to select an alternate route to accommodate the aircraft. To illustrate the proposed integer linear programming model, 5 destination points each with 3 alternate routes and 5 flights underway, are considered. The hypothetical characteristics of the alternate routes in terms of its length and cost in reaching the destination points are illustrated in Figure 3. 
It can be noted that each route is designed to vary in length and cost as is randomly assigned. While alternate routes are set in random for the case of this paper, so is the charge of using a particular route representing only the utilization cost essentially excluding other relative costs to longer flying times. It is further imperative to consider that the length of routes does not necessarily dictate the cost of using the route. That is, a shorter route may not imply the most expensive route to take as charging of routes is dependent on other aspects aside from the distance flown above each member state, for instance [21].

A summary of results from the application of the proposed model to the Lingo software is presented in Table 1. Here, it shows the preferred alternate route of each stakeholder for the given destination points. For brevity purposes, three alternate routes are established for each destination point having different characteristics in terms of length, capacity, and cost of utilization (e.g., alternate routes for Destination 1 are denoted as A, B, and, C). Following the integer linear programming model, a ' 1 ' indicated on the table cell signifies choice of a stakeholder to an alternate route. On the other hand, a ' 0 ' signifies an alternate route that is not selected.

\section{Collaborative Rerouting Decision-Making}

This section describes the rerouting decision arrived from the implementation of the proposed integer linear programming model for a multiple destination case. Since the model aims to satisfy the collaborative interests of stakeholders in the commercial aviation industry, that is, to ensure the safety of operations among flights, it sufficiently includes the individual interests of stakeholders as system constraints under the bracket of expediting air traffic, ensuring efficient use of airspace resources, and achieving economical operation of flights.

For Destination 1, the model selects the shortest route and at the same time most expensive. A similar route characteristic is chosen for Destination 2. As for Destination 3, the route that is moderately expensive and moderately lengthy is selected. In a case of equidistant routes with a slightly similar cost as in Destination 4, the one with the least cost is chosen. On the other hand, in the case of a similarly charged route with varying lengths as in Destination 5 (i.e., one route is shortest in length while the other two are equidistant from the origin to the destination point), the selected route is the longest one. In the case of Destination 6, the shortest route with the least cost is preferred. While Destination 7 selects the longest route with the highest cost. Similarly, for Destination 8, the longest route is also chosen among equally charged alternate routes.

Recall that the parameters of the model (e.g., distance from the origin to the destination point and its corresponding cost when used) are randomly set to represent the realistic conditions in airspace management. Such condition, for instance, displays an expensive route that is independent of its lateral position from each point. Based on these results, two major observations can be made: one, the model, which ensures a safe operation of flights via a safe separation distance between flights, does not select the route that has the shortest length from the origin to the destination point at all conditions. For instance, the model selects the moderately lengthy route for Destination 3 while it selects the longest route for Destination 5, 7, and 8. Furthermore, it can be noted that while the model does not select the shortest route at all times, it also does not select always the least expensive. In fact, in the case given in this paper, out of the eight destination points set with varying cost of alternate routes, four of which selected by the model (i.e., Destination 1, 2, 3, and 5) are not necessarily the least expensive alternative. Such results reflect the collaborative decisionmaking in the event of rerouting flights without prejudice in the individual interests of stakeholders which immediately benefits their respective sectors. 


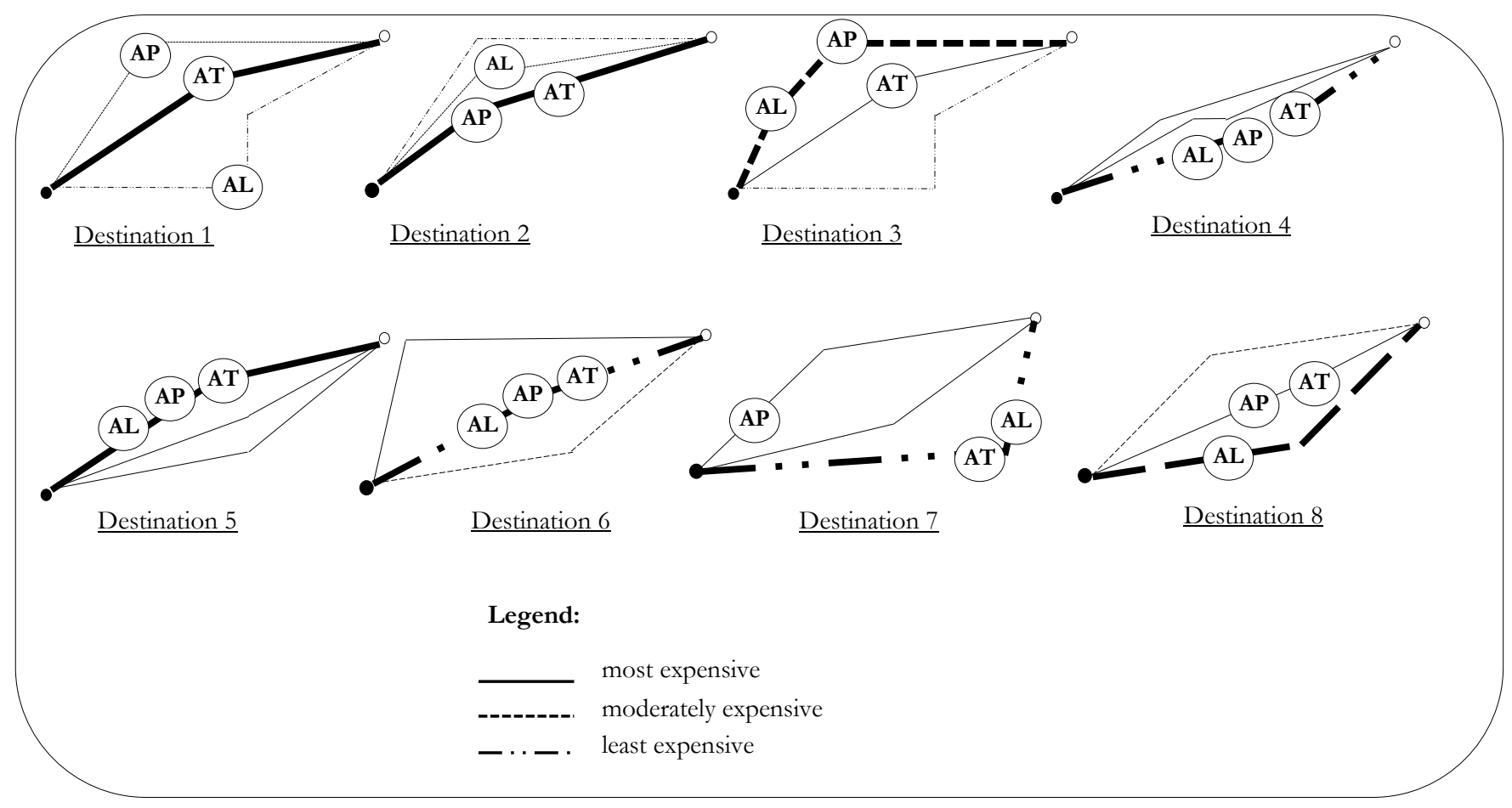

Figure 3. Summary of selected routes with respect to each stakeholder (Note: AP represents the airport management, AL represents the airline sector, and AT represents the air traffic management)

Table 1. Summary of the Proposed Integer Linear Programming Model for Post-Departure Rerouting of Aircraft

\begin{tabular}{|c|c|c|c|c|c|c|c|c|c|c|c|c|c|c|c|c|c|c|c|c|c|c|c|c|}
\hline \multirow{2}{*}{ Stakeholder } & \multicolumn{3}{|c|}{ Destination 1} & \multicolumn{3}{|c|}{ Destination 2} & \multicolumn{3}{|c|}{ Destination 3} & \multicolumn{3}{|c|}{ Destination 4} & \multicolumn{3}{|c|}{ Destination 5} & \multicolumn{3}{|c|}{ Destination 6} & \multicolumn{3}{|c|}{ Destination 7} & \multicolumn{3}{|c|}{ Destination 8} \\
\hline & $\mathbf{A}$ & $\mathbf{B}$ & $\mathbf{C}$ & D & $\mathbf{E}$ & $\mathbf{F}$ & $\mathbf{G}$ & $\mathbf{H}$ & $\mathbf{I}$ & $\mathbf{J}$ & $\mathbf{K}$ & $\mathbf{L}$ & $\mathbf{M}$ & $\mathbf{N}$ & $\mathbf{O}$ & $\mathbf{P}$ & $\mathbf{Q}$ & $\mathbf{R}$ & $\mathbf{S}$ & $\mathbf{T}$ & $\mathbf{U}$ & $\mathbf{V}$ & $\mathbf{W}$ & $\mathbf{X}$ \\
\hline Collaborative DM & 0 & 1 & 0 & 1 & 0 & 0 & 1 & 0 & 0 & 1 & 0 & 0 & 1 & 0 & 0 & 1 & 0 & 0 & 0 & 0 & 1 & 0 & 1 & 0 \\
\hline Airlines & 0 & 0 & 1 & 0 & 1 & 0 & 1 & 0 & 0 & 1 & 0 & 0 & 1 & 0 & 0 & 1 & 0 & 0 & 0 & 0 & 1 & 1 & 0 & 0 \\
\hline Airport management & 1 & 0 & 0 & 1 & 0 & 0 & 1 & 0 & 0 & 1 & 0 & 0 & 1 & 0 & 0 & 1 & 0 & 0 & 1 & 0 & 0 & 1 & 0 & 0 \\
\hline ATC & 0 & 1 & 0 & 1 & 0 & 0 & 0 & 0 & 1 & 1 & 0 & 0 & 1 & 0 & 0 & 1 & 0 & 0 & 0 & 0 & 1 & 1 & 0 & 0 \\
\hline
\end{tabular}


In one particular case, the shortest and least expensive route is selected for Destination 6. Such result satisfactorily represents the interests of all stakeholders given the number of aircraft considered in the case study. A sample solution report from Lingo is shown below.

$\begin{array}{ll}\text { Global optimal solution found. } & \\ \text { Objective value: } & 5.000000 \\ \text { Objective bound: } & 5.000000 \\ \text { Infeasibilities: } & 0.000000 \\ \text { Extended solver steps: } & 0 \\ \text { Total solver iterations: } & 0 \\ \text { Elapsed runtime seconds: } & 0.41 \\ & \\ \text { Model Class: } & \text { PILP } \\ \text { Total variables: } & 15 \\ \text { Nonlinear variables: } & 0 \\ \text { Integer variables: } & 15 \\ & \\ \text { Total constraints: } & 31 \\ \text { Nonlinear constraints: } & 0 \\ & \\ \text { Total nonzeros: } & 75 \\ \text { Nonlinear nonzeros: } & 0\end{array}$

The succeeding sections illustrate the probable changes in the model results should the individual interests of stakeholders are taken in isolation in the rerouting decision without regard to all the other stakeholders' interests.

\section{Rerouting Decision Based on Airlines' Interests}

While the proposed integer linear programming model is designed to consider the collaborative decision-making of stakeholders, this section presents how the rerouting decision considerably varies with the exclusive attention to only the airline sector's interest in delivering flights economically. The proposed model is adjusted to only include this stakeholder's system constraint while maintaining the main objective of ensuring the safe operations of flights.

Note that in both Destination 1 and 2, the model selects an alternate route that is different from that of the initial model's results. Instead of selecting the route that is the shortest and most expensive, here the model returns a route that is the longest and least expensive (see Destination 1). Interestingly, for Destination 2, the model selects the route that is moderately lengthy and moderately expensive instead of the shortest and most expensive route previously chosen. Under cases in Destination 1, 2, 6, and 7, the model never selected the most expensive route as is previously selected in the initial model considering the collaborative decision among stakeholders. It selects either the least expensive or moderately expensive alternate route (see Destination 1,2, 6, 7, and 8) of which direction is likely intuitive. As for Destination 1,2, and 5, no change in the selected route can be noted even with the adjustments made in the model. Even in the isolation of the system constraint representing the cost implications of selecting an alternate route, the model still returns the route that is economical (see Destinations 3, 4, 6 and 7). In Destination 5, however, it is interesting to note that while the alternate routes have the same cost when used and differ 
only slightly with its length, the model tends to select the longest route. A similar choice of route is evident when the collaborative interests of stakeholders are taken into account as in the previous case. In contrast to that, the model selects the shortest route among alternate routes with the same cost (see Destination 8).

Ultimately, the results in this scenario taking the interest of the airline sector alone present two major insights. First, in conditions where the characteristics of alternate routes are found to be significantly varying (i.e., different length and corresponding costs), the chosen routes are never the most expensive ones but those that are economical (i.e., either least expensive or moderately expensive) regardless of its length. In other words, even when the alternate route takes on the longest distance from the origin point to the destination point for as long as it is economically favorable, it is ultimately preferred (see, for instance, Destination 1). Similarly, when alternate routes provide equidistance from the origin point to the destination point but vary in cost, the least expensive one is further selected. As such cases, it is apparent how the cost implications of alternate routes affect the rerouting decision particularly when the interest of the airline sector is taken into consideration. Second, in an instance when the cost of using alternate routes of varying distances is exactly similar, the longest route is still selected. It is rather counterintuitive how, at the same expense, a longer route is preferred over a shorter route.

\section{Rerouting Decision Based on Airport Management's Interests}

In a rerouting decision based on the views of the airport management, the primary goal is hinged toward the efficient use of resources such as airports and airspaces. Considering that the airport management is not at all charged for the use of any resources, in the case of post- departures, airspaces or alternate routes, the main concern of this stakeholder lies on the capacity of the routes to handle traversing flights such that sufficient allocation of resources is observed while maintaining a safe distance among aircraft. In this regard, the proposed model is adjusted to focus on its capacity-related constraint representing the interest of the airport management.

Interestingly, the results obtained are similar to the initial model with the exemption of Destination 1 which selects the route that is moderately expensive and is moderately lengthy. When considering only the interest of the airport management, the model also deviates to that of the collaborative preference of stakeholders by selecting the shortest route (see Destination 7 and 8). Note further that the selection of the shortest route does not necessarily imply less utilization of route as a given number of aircraft is explicitly set. A close association can be further noted with the length of routes and its capacity; that is, a longer route allows for more aircraft to fly at the same time, thus, increasing the minimum separation distance among aircraft. While intuition dictates for the model to select the route that has a higher capacity over other alternatives to ensure maximum separation distance among aircraft, the same is not the case for the adjusted model as it does not prefer long routes in all destinations (see Destination 1, 2, 3, 6, 7, and 8).

Given the number of flights considered in this paper, a longer route does not necessarily guarantee efficient use of routes based on other alternatives available. As an illustration, suppose there are two routes available for use with a capacity of 20 aircraft and 15 aircraft, respectively. While the two routes can cater, say, 10 aircraft at a given period, the longer route appears to be underutilized compared to the shorter one. Therefore, a longer route which in essence has higher capacity may not necessarily be a favorable alternative in terms of efficient resource utilization. However, such a premise does not certainly imply that a shorter route is a better option; instead, the decision relies greatly on the number of aircraft considered at a given point in time, at least as far as the interest of the airport management is concerned. 


\section{Rerouting Decision Based on Air Traffic Management's Interests}

In aircraft operations, the air traffic management division, typically belonging to a separate regulatory body of a country, is responsible for maintaining a safe, orderly, and expeditious flow air traffic between aircraft [35]. During unforeseen instances of, for instance, air traffic congestion due to adverse weather, the rerouting decision depends on the assumed responsibility of this stakeholder by delivering flights in an expeditious manner. Suppose that the speed of aircraft as it traverses from one point to another, as in the case of this paper, remains constant until it approaches the arrival terminal airspace (i.e., way out at the end of the alternate route), the initial choice for an alternate route has to be one that is shortest.

Adjusting the proposed integer linear programming model based on the interest of this stakeholder, it can be noted that the selected routes are closely similar to the initial model consisting of the collaborative decision among stakeholders including the airline sector and airport management. The only difference, though, can be seen on the selected route for Destination 3 and 8. Instead of taking the alternate route that is moderately lengthy and moderately expensive, here, the shortest and most expensive route is chosen. When looked into thoroughly, all the selected routes under this scenario which isolates the interest of the air traffic management as a system constraint referred to the shortest alternate route (see Destination 1, 2, 3, 4, 6, and 8) except for Destination 5 and 6 which selected the longest route. Furthermore, four out of five routes selected are most expensive for all destination points (see Destination 1, 2, 3, and 5). As for Destination 4, while all three alternate routes are equidistant from the origin point to the destination point, the model chose the least expensive one.

\section{Synthesis of the Stakeholder-Specific Decision during Rerouting of Flights}

Evaluating the results of the proposed integer linear programming model as well as its adjusted version according to the individual interests of each stakeholder, interesting insights can be drawn as to how dynamic the results become should the decision be focused on the individual interest of a particular stakeholder alone. It can be observed from the results that an apparent conflict of interests operationally exists in terms of selecting an alternate route during air traffic congestion. A clear manifestation of such conflict can be noted in Destination 1 and 7. Here, the given alternate routes possess various lengths and costs of utilization. As a result of implementing the proposed model with adjustment on the different interests of each stakeholder, it can be noted that all three stakeholders prefer an alternate route that is unique from each other. Due to the complex nature of a rerouting decision during uncertain aircraft situations, the need to reconcile the respective interests among stakeholders becomes dire and even intensified with the sense of urgency it requires. Furthermore, in cases of post-departure operations when flights are already underway to its destination point, sound tactical planning must be arranged from among the stakeholders in such a way that the supposed measure to be taken satisfies all entities concerned.

Another manifestation of conflict in interest among stakeholders can be seen in the results for rerouting in Destination 2. Both airport management and air traffic management choose the shortest and most expensive alternate route in contrast to the choice of the airline sector on a relatively long but moderately expensive route. While the collaborative decision prompts the stakeholders to select the shortest and most expensive route, it appears to satisfy the individual preference of the airport management and air traffic management. The same result can be seen for Destination 2 which ultimately chooses the alternate route that is adherent to the interests of two stakeholders (i.e., airline sector and airport management). As such, the proposed integer linear programming model seems to 
select the route that is preferred by most stakeholders, notwithstanding its characteristics in terms of length and costs as these are particularly embedded as inherent interests of the stakeholders. Furthermore, while one stakeholder's preference does not appear to be satisfied in the proposed model (see, for instance, Destination 2, 3, and 8), the overall goal of ensuring safety by maximizing the separation distance among flights is reached at a level that is satisfactory among stakeholders. Lastly, no conflict of preference is apparent in cases when the alternate routes' characteristics do not vary significantly as in Destinations 5, 6, and 7 which routes' cost is exactly similar to each other and lengths' closely similar. Correspondingly, when the alternate routes are equidistant from the origin point to the destination point as in Destination 4, the proposed model selects a route that is in agreement to the interests of the stakeholders in an expeditious, economical, and capacity-efficient manner.

With such results at hand, it can be inferred that the proposed integer programming model inclines to produce a decision hinged on an alternate route that satisfies the general interests of stakeholders. Although based on the results shown previously, not all stakeholders may agree with the preferred alternate route obtained from the proposed model due to the inherent differences of interests upheld by each. Nevertheless, the proposed model can pool a generalized decision that is refined and richer in a way that an optimum safety level in terms of separation distance among aircraft is achieved. Such a decision, despite its seeming divergence to a stakeholder's preference, ensures that the common goal of stakeholders is reached satisfactorily.

\section{Conclusions}

Weather uncertainties during aircraft operations, especially at en-route phases, call for a prompt and collaborative decision-making process among key stakeholders in the commercial aviation industry. While the most appropriate air traffic management action to be implemented under such circumstances is already identified, a further note on progressing the implementation, that is, selecting an alternate route, becomes an overarching issue confronting the stakeholders. This can be attributed to the differences in interests among stakeholders, of which at times, may even be conflicting. In this paper, an integer linear programming model is proposed for a rerouting problem that satisfactorily converges to the collective interests of all stakeholders. Such a model provides a collaborative type of decision support system incorporating the individual interests of stakeholders as system constraints while laying down the common goal of ensuring safe operations of flights as the objective function.

A hypothetical case study involving multiple destination points, each with multiple alternate routes, is conducted to illustrate the validity of the model. Key results obtained from the model implementation prove that a more refined decision to a rerouting problem under a post-departure case can be generated when the collective interest of stakeholders is taken as a global objective. Such a result can be noted as entirely different from when the individual interests of stakeholders are taken in isolation. Furthermore, empirical evidence is also found on the contextual contention that the individual interests of stakeholders may, in this particular condition involving rerouting of flights in a postdeparture case, conflict with one another. As far as the proposed model for rerouting is concerned, the computational results suggest that it is possible to arrive at a collaborative decision among stakeholders by pooling their interests as system constraints and setting their major goal as an objective function. Through such an arrangement, not only is the individual interests satisfied but so does the general objective. 
The long-term goal of proposing such a model in the air traffic congestion domain is to enable a refined decision-making process during any eventuality involving uncertainties especially in one of the most crucial phases of a flight. As a forward-looking direction, the proposed model can be extended into the robust model such that the solution for any realization of uncertainty (e.g., capacity uncertainties during inclement weather) may be immunized while providing computationally tractable, robust, quality solutions. Furthermore, a wider scope involving pre-departure phases of flights may also be incorporated in the model formulation to capture the bigger picture to whichever instance a disruption may occur needing immediate decision from stakeholders.

\section{References}

[1] J. Yin, M. Hu, Y. Ma, K. Han, and D. Chen, "Airport taxi situation awareness with a macroscopic distribution network analysis," Networks and Spatial Economics, Vol. 19, pp. 669-695.

[2] A.R. Odoni. "The flow management problem in air traffic control," In Flow Control of Congested Networks, A.R. Odoni, L. Bianco, G. Szego, eds.: Springer-Verlag, Berlin, Germany, 1987, pp. 269-288, 1987.

[3] M.F. Bongo, and L.A. Ocampo, "A hybrid fuzzy MCDM approach for mitigating airport congestion: A case in Ninoy Aquino International Airport," Journal of Air Transport Management, Vol. 63, pp. 1-16, 2017.

[4] W. Ding, Y. Zhang, and M. Hansen, "Downstream impact of flight rerouting," Transportation Research Part C, Vol. 88, pp. 176-186, 2018.

[5] X. Diao, and C.H. Chen, "A sequence model for air traffic flow management rerouting problem," Transportation Research Part E, Vol. 110, pp. 15-30, 2018.

[6] T. Bolić, L. Castelli, L. Corolli, and D. Rigonat, "Reducing ATFM delays through strategic flight planning," Transportation Research Part E, Vol. 98, pp. 42-59, 2017.

[7] D. Bertsimas, and S. Stock Patterson, "The air traffic flow management problem with enroute capacities," Operations Research, Vol. 46, No. 3, pp. 406-422, 1998.

[8] D. Bertsimas, and S. Stock Patterson, "The traffic flow management rerouting problem in air traffic control: A dynamic network flow approach," Transportation Science, Vol. 34, No. 3, pp. 239-255, 2000.

[9] D. Bertsimas, G. Lulli, and A. Odoni, "An integer optimization approach to large-scale air traffic flow management," Operations Research, Vol. 59, No. 1, pp. 211-227, 2011.

[10] X. Zhang, M. Xiao, and M. Zhang, "Multi-objective evolutionary algorithm based on decomposition for air traffic flow network rerouting problem," In Intelligent Data Engineering and Automated Learning - IDEAL 2013. IDEAL 2013, Lecture Notes in Computer Science, H. Yin, K. Tang, Y. Gao, F. Klawoon, M. Lee, T. Weise, B. Li, X. Yao, eds.: Springer Berlin, Heidelberg, Vol. 8206, pp. 487-496, 2013.

[11] D. García-Heredia, A. Alonso-Ayuso, and E. Molina, "A Combinatorial model to optimize air traffic flow management problems," Computers and Operations Research, Vol. 112, pp. 104768, 2019.

[12] A. Agustín, A. Alonso-Ayuso, L.F. Escudero, and C. Pizzaro, "On air traffic flow management with rerouting Part I: Deterministic case," European Journal of Operational Research, Vol. 219, pp. 156-166, 2012.

[13] Y. Tian, L. Wan, C.H. Chen, and Y. Yang, "Safety assessment method of performancebased navigation airspace planning," Journal of Traffic and Transportation Engineering, Vol. 2, No. 5, pp. 338-345, 2015.

[14] F. Netjasov, D. Crnogorac, and G. Pavlović, "Potential safety occurrences as indicators of air traffic management safety performance: A network based simulation model," Transportation Research Part C, Vol. 102, pp. 490-508, 2019. 
[15] N. Kalemba, and F. Campa-Planas, "Safety and the economic and financial performance in the airline industry: Is there any relationship?," Aviation, Vol. 23, No. 1, pp. 7-14, 2019.

[16] M. Sauer, M. Steiner, R.D. Sharman, J.O. Pinto, and W.K. Deierling, "Tradeoffs for routing flights in view of multiple weather hazards," Journal of Air Transportation, Vol. 27, No. 2, pp. 70-80, 2019.

[17] T. Andersson Granberg, and A. Oquillas Munoz, "Developing key performance indicators for airports", In: $3^{\text {rd }}$ ENRI International Workshop on ATM/CNS, Tokyo, Japan, 2013.

[18] W. Jinyu, and Y. Xiaohuan, "Airline safety risk evaluation based on factor analysis," Advanced Materials Research, Vol. 774-776, pp. 1940-1944, 2013.

[19] W. Chen, and J. Li, "Safety performance monitoring and measurement of civil aviation unit," Journal of Air Transport Management, Vol. 57, pp. 228-233, 2016.

[20] R. Arnaldo Valdés, V.F.G. Comendador, J.A.P. Castan, A.R. Sanz, L.P. Sanz, F.J.S. Nieto, and E.S. Aira, "Bayesian inference in Safety Compliance Assessment under conditions of uncertainty for ANS providers," Safety Science, Vol. 116, pp. 183-195, 2019.

[21] J. Rosenow, and H. Fricke, "Impact of multi-criteria optimized trajectories on European airline efficiency, safety and airspace demand," Journal of Air Transport Management, Vol. 78, pp. 133-143, 2019.

[22] M. Ellejmi, M. Makhouf, J. Toussaint, and I. de Visscher, "Safety studies of reducing minimum radar separation for high performing airport operations," In: 2019 Integrated Communications, Navigation and Surveillance Conference (ICNS), Herndon, Virginia, United States of America, pp. 1-10, 2019.

[23] G. Di Gravio, M. Mancini, R. Patriarca, and F. Costantino, "Overall safety performance of the air traffic management system: Indicators and analysis," Journal of Air Transportation Management, Vol. 44-45, pp. 65-69, 2015.

[24] M.F. Bongo, and L.A. Ocampo, "Exploring critical attributes during air traffic congestion with a fuzzy DEMATEL-ANP technique: A case study in Ninoy Aquino International Airport," Journal of Modern Transportation, Vol. 26, pp. 147-161, 2018.

[25] S. Kaspers, N. Karanikas, A. Roelen, S. Piric, and R.J. de Boer, "How does aviation industry measure safety performance? Current practice and limitations," International Journal of Aviation Management, Vol. 4, No. 3, pp. 224-245, 2019.

[26] International Civil Aviation Organization, Asia/Pacific Regional: Air Traffic Flow Management Concept of Operations, ICAO Asia and Pacific Office, Bangkok, Thailand, 2015.

[27] M. Janic, "A multi-criteria evaluation of solutions and alternatives for matching capacity to demand in an airport system: the case of London," Transportation Planning and Technology, Vol. 38, No. 7, pp. 709-737, 2015.

[28] P. Belobaba, A.R. Odoni, and C. Barnhart, The Global Airline Industry, ${ }^{\text {nd }}$ Edition, Wiley, Hoboken, New Jersey, United States of America, 2015.

[29] M. Arblaster, "Chapter 8 - Economic regulation of air traffic management: The structure of charges," Air Traffic Management. Economics, Regulations and Governance, pp. 173-189, 2018.

[30] K.G. Zogafros, M.A. Madas, and Y. Salouras, "A decision support system for total airport operations management and planning," Journal of Advanced Transportation, Vol. 47, pp. 170-189, 2013.

[31] S.E. Stalnaker, J.S. DeArmon, and R.D. Katkin, "Collaborative airspace congestion resolution (CACR) benefits analysis," In: 2009 IEEE/AIAA $28^{\text {th }}$ Digital Avionics Systems Conference, Orlando, Florida, pp. 3.C.2-1-3. C.2-7, 2009. 
[32] C.R.M. Murça, and R.J. Hansman "Predicting and planning airport acceptance rates in metroplex systems for improved traffic flow management decision support," Transportation Research Part C, Vol. 97, pp. 301-323, 2018.

[33] M. Samà, A. D'Ariano, F. Corman, and D. Pacciarelli, "Coordination of scheduling decisions in the management of airport airspace and taxiway operations," Transportation Research Part A, Vol. 114, pp. 398-411, 2018.

[34] T.G. Reynolds, "Air traffic management performance assessment using flight inefficiency metrics," Journal on Transport Policy, Vol. 34, pp. 63-74, 2014.

[35] G. Tobaruela, P. Fransen, W. Schuster, W.Y. Ochieng, and A. Majumdar, "Air traffic predictability framework development, performance evaluation and application," Journal of Air Transport Management, Vol. 39, pp. 48-58, 2014.

[36] M. Samà, A. D'Ariano, F. Corman, and D. Pacciarelli, D., "Coordination of scheduling decisions in the management of airport airspace and taxiway operations," Transportation Research Procedia, 22nd International Symposium on Transportation and Traffic Theory, Vol. 23, pp. 246-262, 2017.

[37] T. Pejovic, R.B. Noland, V. Williams, and R. Toumi, "A tentative analysis of the impacts of an airport closure," Journal of Air Transport Management, Vol. 15, pp. 241248, 2009.

[38] Y. Hu, H. Liao, S. Zhang, and Y. Song, "Multiple objective solution approaches for aircraft rerouting under the disruption of multi-aircraft," Expert Systems with Applications, Vol. 83, pp. 283-299, 2017.

[39] D. Zhang, H.H. Lau, and C. Yu, "A two stage heuristic algorithm for the integrated aircraft and crew schedule recovery problems," Computers \& Industrial Engineering, Vol. 87, pp. 436-453, 2015.

[40] E.K. Burke, P. De Causmaecker, G. De Maere, J. Mulder, M. Paelinck, and G. Vanden Beghe, "A multi-objective approach for robust airline scheduling," Computers \& Operations Research, Vol. 37, pp. 822-832, 2010.

[41] The International Air Transport Association (IATA), Principles of Airport Handling, $1^{\text {st }}$ Edition, Montreal, Quebec, Canada, 1980.

[42] M.A.A. Makhloof, M.E. Waheed, and U.A.E.R. Badawi, "Real-time aircraft turnaround operations manager," Production Planning \& Control: The Management of Operations, Vol. 25, No. 1, pp. 2-25, 2014.

[43] V. Kharchenko, and K. Tapia, "Analysis of aircraft delays at the stage of arrival at airport," Proceedings of the National Aviation University, Vol. 3, No. 60, pp. 28-32, 2014.

[44] C. Barnhart, and V. Vaze, "Modeling airline frequency competition for airport congestion mitigation," Transportation Science. Institute for Operations Research and the Management Sciences, Vol. 46, No. 4, pp. 512-535, 2012.

[45] L. Adacher, and M. Flamini, "Air robust routes: Analysis of the direct costs," IFAC PapersOnLine, Vol. 51-7, pp. 70-75, 2018.

[46] M.C.R. Murça, "Collaborative air traffic flow management: Incorporating airline preferences in rerouting decisions," Journal of Air Transport Management, Vol. 71, pp. 97-107, 2018. 